\title{
RESOLUTIVIDADE E FERRAMENTAS PARA CUIDAR: UM ESTUDO COM MULHERES QUE VIVEM COM CÂNCER DE MAMA
}

RESOLUTIVENESS AND TOOLS TO CARE: A STUDY WITH WOMEN LIVING WITH BREAST CANCER

RESOLUCIÓN Y HERRAMIENTAS PARA CUIDAR: UN ESTUDIO CON MUJERES QUE VIVEN COM CÁNCER DE MAMA

Marcio Costa de Souza

Juliana Costa dos Santos Borges 2

Kamila Freitas Trindade ${ }^{3}$

Bruna Pereira Neves ${ }^{4}$

Evelin Duarte Serpa ${ }^{5}$

Milene Pereira de Souza Santos 6

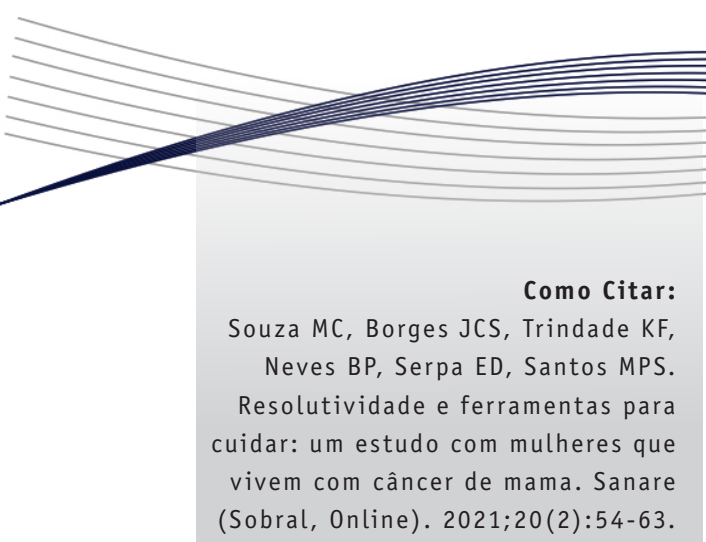

(Sobral, Online). 2021;20(2):54-63.

Descritores:

Neoplasias da mama; Acesso aos serviços de saúde; Continuidade da assistência ao paciente.

Descriptors: Breast neoplasms; Access to health services; Continuity of patient care.

Descriptores: Neoplasias de la mama; Acceso a los servicios de salud; Continuidad de la asistencia al paciente.

Submetido: $16 / 10 / 2021$

Aprovado:

24/11/2021

Autor(a) para Correspondência: Marcio Costa de Souza Universidade Estadual da Bahia Endereço: Rua Silveira Martins, 2555, Cabula, Salvador, Bahia CEP:41150-000 E-mail:mcsouzafisio@gmail.com

\section{RESUMO}

Este artigo objetiva conhecer as ferramentas e a resolutividade do cuidado com mulheres que vivem com câncer de mama. Optou-se pela abordagem qualitativa de caráter exploratório e utilizou-se como técnica para a produção dos dados a entrevista semiestruturada, que teve a participação de 11 mulheres, e a amostra foi definida por saturação das respostas. A pesquisa foi realizada em um centro de referência estadual em oncologia. Para a interpretação dos dados, a análise de conteúdo foi escolhida. No que tange à resolutividade, foi observado que há barreiras de acesso e estas produzem iniquidades, muitas vezes resolvidas a partir de redes vivas de existência estabelecidas pelas mulheres que vivem com câncer de mama. Como ferramentas para a resolutividade do cuidar, operam a necessidade de utilização de tecnologias do cuidado, além do aprimoramento dos fluxos de atenção à saúde construídos. Portanto, a produção do cuidado com o olhar subjetivo configura-se como estratégia fundamental para o alcance da resolutividade em saúde de mulheres acometidas pelo câncer de mama por meio das tecnologias leves e do reconhecimento das redes e tessituras no cotidiano da vida dessas pessoas.

\footnotetext{
1. Fisioterapeuta. Mestre em Saúde Coletiva. Universidade Estadual da Bahia. Departamento de Ciências da Vida. E-mail: mcsouzafisio@gmail.com 0RCID: http://orcid.org/0000-0002-4922-6786

2. Graduanda em Nutrição da Universidade Estadual da Bahia. Departamento de Ciências da Vida. E-mail: jucsborges@gmail.com ORCID: http://orcid.org/0000-0002-5711-8136

3. Graduanda em Medicina da Universidade Estadual da Bahia. Departamento de Ciências da Vida. E-mail: freitastmila@gmail.com 0RCID: http://orcid.org/0000-0003-1603-8196

4. Graduanda em Medicina da Universidade Estadual da Bahia. Departamento de Ciências da Vida. E-mail: brunaneves212120@gmail.com 0RCID: http://orcid.org/0000-0003-3191-2419

5. Graduanda em Medicina da Universidade Estadual da Bahia. Departamento de Ciências da Vida. E-mail: dserpa99@ gmail.com ORCID: http://orcid.org/0000-0002-0907-1572

6. Enfermeira. Mestra em Saúde Coletiva. UniDomBosco. E-mail: profmilenescmed@gmail.com ORCID: http://orcid. org/0000-0003-1278-5012
} 


\section{ABSTRACT}

This article aims to know the tools and the resoluteness of care for women living with breast cancer. A qualitative exploratory approach was chosen, and a semi-structured interview was used as a technique for data production with the participation of 11 women and a sample defined by response saturation. The research was carried out at a state referral center for oncology. Content analysis was chosen for data interpretation. As regards for resoluteness, we observed that there are access barriers and these produce inequities, often resolved from living networks of existence established by women living with breast cancer. As tools for the resoluteness of care, there is the need to use care technologies in addition to the improvement of constructed healthcare flows. Therefore, the production of care with a subjective look is configured as a fundamental strategy for achieving health resolution for women suffering from breast cancer through light technologies, and the recognition of networks, and the fabric of these people's daily lives.

\section{RESUMEN}

Este artículo tiene como objetivo conocer las herramientas y la resolución del cuidado con mujeres que viven con cáncer de mama. Se ha elegido el abordaje cualitativo de carácter exploratorio y se utilizó como técnica para la producción de los datos la entrevista semiestructurada, que tuvo la participación de 11 mujeres y la amuestra fue definida por saturación de las respuestas. La investigación fue realizada en un centro de referencia estadual en oncología. Para la interpretación de los datos, el análisis de contenido fue elegida. En lo que toca la resolución, fue observado que hay barreras de acceso y estas producen iniquidades, muchas veces resueltas a partir de redes vivas de existencia establecidas por las mujeres que viven con cáncer de mama. Como herramientas para la resolución del cuidar, operan la necesidad de utilización de tecnologías del cuidado, además del mejoramiento de los flujos de atención a la salud construidos. Por lo tanto, la producción del cuidado con la mirada subjetiva se configura como estrategia fundamental para lograr la resolución en salud de mujeres acometidas por el cáncer de mama por medio de las tecnologías leves y del reconocimiento de las redes y tesituras en el cotidiano de la vida de esas personas.

\section{INTRODUÇÃ 0}

0 câncer de mama configura-se, notoriamente, como um dos principais problemas de saúde pública mundial ${ }^{1}$. De acordo com o Global Câncer Observatory da International Agency for Research on Cancer, as taxas estimadas de incidência para o ano de 2020, em todo o mundo, para ambos os sexos e em todas as idades, demonstraram que o câncer de mama é o tipo de neoplasia com maior incidência em âmbito mundial, representando cerca de $47,8 \%$ dos casos analisados ${ }^{2}$. No Brasil, com base nos dados do Instituto Nacional de Câncer José Alencar Gomes da Silva, estima-se que ocorrerão 66 mil novos casos de câncer de mama no país para cada ano do triênio 2020-2022 ${ }^{1}$. Tais dados reforçam e enfatizam ainda mais a necessidade de atenção por parte dos serviços de saúde a este crescente público.

No que concerne à sua tipicidade, é de conhecimento universal que o câncer de mama é uma doença caracteristicamente heterogênea e, assim, o tratamento varia em função de critérios como agressividade do tumor, subtipo molecular das células cancerígenas, mutações, entre outros. Postula-se, hoje, que o principal tratamento para a cura do câncer é a cirurgia, porém tratamentos adjuvantes como a quimioterapia, a radioterapia, a terapia hormonal e a imunoterapia reduzem o risco de recidiva do câncer de mama e, consequentemente, o risco de morte por essa doença ${ }^{3}$. No entanto, a literatura tem mostrado que a resolução das necessidades em saúde dos indivíduos acometidos por essa patologia não se limita apenas ao tratamento medicamentoso e cirúrgico ${ }^{4}$.

Isso porque, ao analisarmos os impactos que 0 câncer de mama pode causar na vida da população diagnosticada, composta majoritariamente pelo público feminino, é perceptível que as repercussões perpassam os danos fisiológicos ${ }^{5,6}$. Mulheres em tratamento em decorrência de neoplasia mamária enfrentam uma variedade de situações potencialmente estressantes, gerando sérias repercussões não só físicas, como também psicológicas?. Sentimentos como medo, angústia, desesperança, dentre outros, são marcantes no cotidiano da mulher com câncer de mama, o que leva 
ao isolamento social e familiar, proporcionando, consequentemente, a perda de sua identidade diante da sociedade 5 . Destarte, compreende-se que para que haja uma maior resolutividade das demandas em saúde, é necessária uma atenção à saúde que busque a efetividade, e que assim possa atender às suas necessidades subjetivas ${ }^{8}$.

Diante deste contexto, é importante salientar sobre o conceito de resolutividade em saúde, que pode ser definido como uma resposta satisfatória ofertada pelo serviço à usuária que busca atendimento com o intuito de suprir as suas necessidades em saúde9. Porém, o conceito de resolutividade não engloba apenas a cura da doença como objetivo final, mas, também, a garantia da redução do sofrimento, bem como a promoção e a manutenção da saúde e, por consequência, uma melhor qualidade de vida ${ }^{10}$.

Não obstante, configura-se de extrema importância a análise da produção de um cuidado resolutivo no serviço de saúde dentro da perspectiva da enfermidade e suas múltiplas repercussões. Dada a relevância, objetiva-se, com este estudo, conhecer as ferramentas e a resolutividade do cuidado em mulheres que vivem com câncer de mama.

\section{METODOLOGIA}

0 delineamento metodológico escolhido para a construção da pesquisa foi a abordagem qualitativa, de caráter exploratório. A pesquisa foi realizada no Centro Estadual de Oncologia localizado no município de Salvador, estado da Bahia. No que se refere aos participantes da pesquisa, foram escolhidas mulheres que já se encontravam diagnosticadas e/ou em qualquer fase de tratamento do câncer de mama. Dessa forma, optou-se como critério de inclusão mulheres que se encontravam em tratamento do câncer de mama no serviço de referência estadual escolhido de forma intencional. Como critérios de exclusão, foram excluídas aquelas que no momento da pesquisa estavam realizando tratamento concomitante em outra instituição.

0 processo de produção de dados findou-se com a saturação das informações obtidas. Conforme Freitas et al. ${ }^{11}$, considera-se saturada a coleta de dados quando nenhum novo elemento é encontrado e o acréscimo de novas informações deixa de ser necessário, pois não altera a compreensão do fenômeno estudado. De modo congruente a essa afirmativa, o quantitativo total de 11 entrevistas apresentou-se suficiente em decorrência do não aparecimento de novas informações, gerando uma repetição de dados.

Para a coleta de dados, foram aplicadas entrevistas, norteadas por um roteiro semiestruturado que continha eixos estruturantes: itinerários, resolutividade, redes de cuidado e cuidado em saúde. As perguntas foram realizadas em locais que garantissem o sigilo e foram gravadas em um aparelho Motorola E6s. Na apresentação dos resultados, as participantes são identificadas com o termo Ent. e um número individualizado (Ent. 1, Ent. 2, Ent. 3, etc.).

$\mathrm{Na}$ análise dos dados, foram realizadas as seguintes etapas: transcrição das gravações, organização e leitura do material. Por conseguinte, realizou-se a classificação das informações a partir da identificação dos núcleos de sentidos e, por fim, elaboraram-se as chamadas categorias empíricas para uma análise final dialogando com a teoria já produzida sobre a temática. As categorias empíricas produzidas e suas respectivas subcategorias foram: 1) Barreiras de acesso à resolutividade (barreiras e redes vivas) e 2) Ferramentas para a resolutividade na rede de cuidado (Tecnologias do cuidar, Fluxos/ caminhos do cuidado, Processo de subjetivação da doença e o impacto na resolutividade; Usuária como participante ativa em busca da resolutividade).

Para a análise final das informações, foram realizadas leituras exploratórias e correlações da literatura científica e referencial teórico com a prática e os fatos experienciados. Houve, assim, a articulação dos dados empíricos com os conteúdos teóricos da pesquisa, o que promoveu a relação entre a teoria e a prática. Consequentemente ocorreram deduções das informações colhidas e, simultaneamente, análise do contexto que originou tais informações, com vistas a responder à questão norteadora.

A pesquisa foi aprovada pelo Comitê de Ética em Pesquisa da Universidade do Estado da Bahia, e somente foi iniciada após a concordância dos sujeitos por meio da assinatura do Termo de Consentimento Livre e Esclarecido (TCLE), no ano de 2020, sob o parecer de n. ${ }^{0} 26049219.7 .0000 .0057$.

\section{RESULTADOS E DISCUSSÃO}

\section{Barreiras de acesso à resolutividade}

É fundamental conceituar as barreiras de acesso 
que se conectam de forma potente à resolutividade; sendo assim, estas podem ser compreendidas como todo e qualquer entrave que impossibilite ou dificulte a satisfação das necessidades do usuário por parte do serviço de saúde e que agem de forma sinérgica por efeitos múltiplos ${ }^{12,13}$. A compreensão de tais necessidades requer capacidade de escuta, respeito à diversidade humana, cultural e social e, ainda, compreensão para além do processo saúde e doença ${ }^{14}$. Concomitantemente, caso não haja esse efetivo acolhimento por parte do serviço, poderá ocorrer a criação de novos espaços de cuidado que perpassam a estrutura formal, denominados de redes vivas ${ }^{15}$. Tanto a temática sobre as barreiras de acesso à resolutividade como as redes vivas serão aprofundadas nas subcategorias a seguir.

As barreiras de acesso à saúde podem ser subdivididas em quatro dimensões, sendo elas: acesso econômico, acesso geográfico, acesso funcional e acesso comunicacional. A barreira de acesso que corresponde à dimensão econômica ocorre quando o usuário tem o seu serviço dificultado ou interrompido devido aos custos gerados em busca da assistência. Já a barreira de acesso no âmbito da dimensão geográfica ocorre quando o usuário passa por dificuldades no seu deslocamento para a utilização do serviço, devido à distância e custos de locomoção. No que tange à dimensão funcional, está diretamente relacionada às dificuldades que o usuário enfrenta para adentrar o serviço de saúde propriamente dito. E, por fim, a dimensão comunicacional está ligada aos entraves encontrados pelo usuário no que se refere ao recebimento de informações necessárias (por parte dos trabalhadores que compõem o serviço) para atender às suas demandas em saúde ${ }^{12}$.

As barreiras de acesso foram visivelmente apreendidas em meio às falas das entrevistadas:

[...] demorou, para mim, assim refazer de novo, né? Aí eu saí de Canavieiras e vim fazer em Ilhéus (Ent. 1).

[...] eu acho que eu faria diferente, eu não ia ao posto de saúde, se eu pudesse eu viria logo pra cá, entendeu? Porque no posto de saúde lá foi uma perca de tempo, de certa forma, porque eu ainda fiquei umas duas semanas tentando ir lá [...], isso ainda em abril, depois da Semana Santa eu fiquei ainda umas duas semanas tentando ir lá (Ent. 11).
0 acesso aos serviços de saúde nada mais é do que a capacidade que as pessoas possuem de adentrar e/ ou utilizar o serviço de saúde no momento em que compreendem ser necessário ${ }^{16}$. Ao analisar as falas supracitadas, denota-se a existência de diversos obstáculos no acesso aos serviços de saúde por parte das mulheres com câncer de mama, o que contribui expressivamente para um prognóstico desqualificado para essas usuárias ${ }^{17,18}$. A fala a seguir é uma importante representação das graves consequências para a sobrevida das mulheres que se deparam com essas barreiras no acesso ao serviço:

[...] depois de um ano e pouco, mais de dois anos, um ano e pouco, aí ela me pediu uma tomografia, aí foi que tinha dado no fígado (Ent. 2).

Dessa forma, apesarde a universalidade e a igualdade de acesso serem direitos constitucionalmente instituídos, na prática, ainda se observam inúmeros entraves para a sua operacionalização, tendo como consequência o agravamento do quadro apresentado, o que pode levar ao aumento das incapacidades e/ ou à morte ${ }^{16}$.

Como dito anteriormente, cada ser possui as suas necessidades em saúde, as quais são vividas e experimentadas de forma singular ${ }^{19}$. E é justamente nesse processo de busca por resolutividade que o usuário constrói uma trajetória na rede, considerando suas condições sociais, culturais e o próprio acesso aos serviços, o que muitas vezes não é suprido pela rede formal, surgindo as redes vivas ${ }^{14}$. Assim, as redes vivas são formadas justamente pela incipiência na formação de redes formais capazes de acolher e resolver as necessidades dos usuários ${ }^{19}$.

Pode-se afirmar que as redes vivas são fluxos dinâmicos e mutáveis que surgem de acordo com a singularidade das necessidades de saúde do usuário ${ }^{19}$. A seguir, observa-se na fala da entrevistada que ela utilizou das redes vivas para conseguir antecipar o recebimento do resultado do seu exame,

- resultado da biópsia demora muito pra sair [...], uma coisa que é urgente. Eu só consegui esse resultado dia 15 de janeiro, porque eu tive uma pessoa, que ligou para o laboratório, deu o CRM dela de médica, e conseguiu que o meu resultado saísse em 30 dias (Ent. 10). 
Tais falas reacendem um debate sobre a importância das redes vivas no cuidado em saúde ${ }^{15}$. Isso porque a forma de atuação reproduzida na maioria dos serviços de saúde opera ainda em cima do campo das profissões, procedimentos e protocolos, ou seja, uma lógica burocrática e mecanizada ${ }^{19}$. Dessa forma, faz-se necessário acreditar na potencialidade da rede como algo vivo que proporciona a continuidade do cuidado, pelo fato de estar mais próxima do cotidiano e realidade das pessoas, acolhendo, assim, a diversidade inerente ao ser vivente e à própria sociedade que o cerca ${ }^{15}$. Nas falas transcritas a seguir é perceptível a importância da rede viva no processo saúde-doença das usuárias,

[...] Desde quando meu marido também era motorista da ambulância, aí sempre Dra. G viajava com ele, então Dra. G foi quem mandou me chamar para explicar tudo (Ent. 2).

[...] Como eu trabalho em São Francisco e conheço o pessoal lá da secretaria de saúde... Aí eu pedi pra conseguir fazer minha USG lá. Nisso, eu consegui marcar... a do abdome e a mamária (Ent. 11).

Sendo assim, conhecer alguém ou um meio que viabilize o acesso contribui significativamente para uma melhor resolutividade. Contudo, apesar dessas possibilidades, as redes vivas não configuram uma fixa garantia de que as pessoas terão o acesso no momento em que precisam ${ }^{14}$. Isso acontece porque as redes vivas são fragmentárias e em acontecimento, hipertextuais, ou seja, às vezes são circunstanciais, montam e desmontam, e, às vezes, elas tornam-se mais estáveis ${ }^{19}$.

\section{Ferramentas para a resolutividade na rede de cuidado}

Cuidar, uma palavra complexa em significado, pode ser entendida como um ato de ocupação, preocupação, responsabilização e envolvimento, exigindo do cuidador conhecimento, sensibilidade e um estado de disponibilidade para o outro ${ }^{8}$. Quando um ser vivente perpassa por um processo de adoecimento, um dos requisitos necessários para se efetivar o cuidado é o seu acesso à saúde, como já discutido anteriormente. Isso significa dizer que, para que haja uma efetiva atenção, não basta que o usuário adentre o serviço, mas que o serviço ofertado seja resolutivo quanto à sua demanda, o que se apresenta como um dos princípios organizativos do Sistema Único de Saúde (SUS) ${ }^{20}$.

É importante destacar que a resolutividade deve abranger problemas de saúde das pessoas que vivem em situação de vulnerabilidade social e biológica ${ }^{21}$. No contexto da mulher com câncer de mama, em decorrência do sofrimento causado pelo tratamento e pelo estigma que a doença carrega, ela acaba por vivenciar, de maneira acentuada, essa realidade, que pode exacerbar quando esta apresenta uma linha de cuidado desarticulada e, consequentemente, pouco resolutiva ${ }^{4,8}$.

Sendo assim, o principal desafio a ser enfrentado é a garantia da continuidade do cuidado nos diversos pontos da rede de serviços, através de mecanismos que visem a uma maior resolutividade em saúde, com uma oferta integral de tecnologias moduladas de acordo com as demandas e necessidades de cuidados singulares, em que se deve considerar a prática interprofissional e colaborativa ${ }^{22}$.

Nos serviços de saúde, a organização do processo de trabalho operando de forma centrada no usuário, de maneira integrada e com ações interprofissionais, ainda configura um dos grandes desafios contemporâneos a serem enfrentados pelos trabalhadores e gestores implicados na produção do cuidado $^{22}$. A busca objetiva do problema biológico (modelo biomédico) leva à prática de ações centradas nos procedimentos, esvaziada de interesse no outro, com escuta empobrecida, gerando, assim, uma grande dificuldade em produzir um cuidado que perpasse a ênfase na materialidade do corpo humano ${ }^{23}$.

Dessa forma, o cuidado é um somatório da articulação de trabalhadores e ambientes e as escolhas quanto à aplicação de tecnologias (dura, leve-dura e leve), em um determinado tempo e espaço, que devem ser utilizadas no processo de trabalho em saúde ${ }^{24}$. Em resumo, as tecnologias duras estão relacionadas à utilização de instrumentos, normas e equipamentos tecnológicos, ou seja, os recursos materiais; já as leve-duras estão relacionadas à construção do conhecimento por meio de saberes estruturados (teorias, modelos de cuidado); e, por fim, as tecnologias leves estão relacionadas à constituição de relações para a implementação do cuidado (vínculo, autonomização, gestão de serviços e acolhimento) ${ }^{25}$.

No que concerne à realidade das mulheres diagnosticadas com câncer de mama, é inegável 
afirmar que a mulher acometida por essa doença enfrenta não apenas alterações físicas em consequência das intervenções, o que produz um comprometimento na sua imagem corporal, na vida social e nos aspectos que envolvem a afetividade ${ }^{4}$. Dessa forma, podemos destacar a relevância do uso das tecnologias leves por parte do serviço na produção do cuidado, como aponta o discurso:

[...] depois que iniciaram esses trabalhos de humanização nos hospitais, eu achei que tudo melhorou, porque a gente consegue ver que, desde a recepção aos atendentes, a técnicos e médicos, e te olham agora na consulta, né? Porque antes a gente não via (Ent. 10).

Apesar da evidente importância do uso dessas tecnologias na rotina do serviço, ainda há uma dificuldade por parte dos trabalhadores de saúde em legitimar e reconhecer essa ferramenta como um instrumento do seu trabalho ${ }^{26}$. Tal realidade compromete diretamente as relações intersubjetivas entre os trabalhadores de saúde e as usuárias, pois essa visão interfere na possibilidade de ofertar uma atenção humanizada e, consequentemente, acolhedora, sem produção de vínculos, o que dificulta a participação ativa dessas mulheres no tratamento e aumenta as barreiras relacionais essenciais para a comunicação e efetivação do cuidado ${ }^{27}$.

Quando discutimos no âmbito da mulher com câncer de mama, é salutar o uso das tecnologias leves, e se estas não fazem parte da caixa de ferramentas, pode-se gerar consequências ainda mais acentuadas devido à fragilidade gerada pela enfermidade e revela a necessidade da humanização em saúde como política, o que pode ser observado abaixo,

[...] aqui, logo no início, quando eu cheguei aqui eu não tive vontade de voltar aqui, não. $\mathrm{Na}$ médica, é porque ela é direta! Quando eu cheguei aqui, eu não sabia de tudo, mas o jeito de falar me entristeceu, [...] do jeito que ela falou... se tivesse metástase em outras partes, aquilo me doeu! Aí, eu disse, 'não vou voltar aqui mais, não! Eu vou morrer em casa!' (Ent. 8).

Diante do exposto, nota-se que nas falas é apresentada uma relação assimétrica e sem cuidado no diálogo do trabalhador com a usuária, colocandose em pauta a necessidade de discutir o modo no qual os profissionais se relacionam no ato de cuidar. Essa análise é atravessada pelos processos formativos, desde as escolas, formação, até as atividades de ensino no cotidiano, o que inclui o debate de instituir práticas humanizadas no cuidado em saúde ${ }^{23}$. Nesse sentido, é fundamental que a formação do trabalhador em saúde possa ter entre seus eixos estruturantes a integralidade, com o propósito de colocar na discussão o cuidado em uma perspectiva subjetiva e com destaque nas necessidades da usuária, com a valorização da autonomia, da realidade social e afetiva de cada sujeito ${ }^{8}$.

Vale salientar que, ao afirmar a necessidade de reconhecer a legitimidade das tecnologias leves como ferramenta indispensável para a produção do cuidado no serviço, não se exclui a importância das demais tecnologias. Ou seja, produzir o cuidado em mulheres com câncer de mama é utilizar de forma conjunta os diversos e importantes aspectos das tecnologias, porém, com o devido enfoque para as tecnologias leves, as quais, na maioria das vezes, possuem um impacto direto e principal sobre a vida e o viver dessas mulheres ${ }^{27}$.

Com a instituição da Política Nacional para a Prevenção e Controle do Câncer na Rede de Atenção à Saúde das Pessoas com Doenças Crônicas no âmbito do SUS, estabelece-se um fluxo de forma regulada e ordenada de usuárias entre os pontos de atenção à saúde, vislumbrando-se práticas resolutivas e orientadas pelo princípio da integralidade. No que se refere às mulheres com câncer de mama, um sistema de referência e contrarreferência eficaz é extremamente essencial para um diagnóstico e tratamento precoces, o que contribui diretamente para um melhor prognóstico ${ }^{28,29}$. As entrevistadas expuseram essa importância:

[...] aí eu fiz a ressonância magnética lá no Hospital do Subúrbio, não foi pago, foi daqui mesmo que elas me encaminharam aqui para o Hospital do Subúrbio (Ent. 3).

[...] aí me encaminhou lá pra secretaria de saúde de São Francisco, para eles entrarem com um pedido pra eu vim diretamente pro Centro de Referência realizar essa triagem pra fazer essa biópsia, tudo pelo SUS (Ent. 11). 
É perceptível a importância da articulação entre os níveis de complexidade para a vida das pacientes. Ao falar de referência e contrarreferência, estamos nos referindo às redes formais para o acesso à saúde, também chamada de Rede de Atenção à Saúde, diferentemente das redes vivas, que são fluxos de acesso à saúde construídos pelas usuárias, que perpassam os muros da rede formal ${ }^{14}$.

Podemos definir a Rede de Atenção à Saúde como arranjos organizativos de ações e serviços de saúde, de diferentes densidades tecnológicas e interdependentes, fundamentais para a garantia do acesso universal da população à saúde com equidade ${ }^{28,29}$. Segundo Paula ${ }^{28}$, a Rede de Atenção ao Câncer de Mama, em específico, só funciona se houver usuárias, políticos, gestores e trabalhadores comprometidos, sendo esta uma condição indispensável para a atenção aos agravos decorrentes de câncer de mama que, por sua natureza, envolvem a Atenção Básica, média e alta complexidade, em conjunto com as estruturas específicas do campo da oncologia. No entanto, nem sempre essa articulação é harmônica, o que pode ser percebido nas falas a seguir:

[...] eu fui ao consultório lá, numa clínica que tem lá, particular, e fiz! Eu fui à rede SUS, mas eu não consegui! (Ent. 8).

[...] eu liguei várias vezes pra regulação, fui às prefeituras bairro e, a minha busca, a minha procura, não tem vaga, não tem vaga. Para você conseguir vaga você tem que esperar assim, não tem nem data pra longe assim, eles não marcam porque é regulado (Ent. 10).

Percebe-se que as usuárias não conseguem um acesso adequado ao sistema de referência e contrarreferência, o qual utiliza de outros meios, para além dos ditos, que estão nas normativas, o que pode, em alguns casos, gerar custos e retardar ainda mais o tratamento, situação que pode produzir itinerários complexos não lineares ${ }^{8}$. De acordo com Goldman ${ }^{29}$, juntamente com a análise das entrevistas realizadas neste estudo, afirma-se que os principais motivos que dificultaram essa integração na Rede de Atenção Oncológica são: fragilidade do vínculo por parte dos trabalhadores de saúde com relação às usuárias, ausência de padronização do sistema de referência e contrarreferência e baixa cooperação entre serviços de saúde municipais e estaduais, o que dificulta para o prognóstico dessas usuárias.

Como dito anteriormente, é inegável afirmar que o câncer de mama é uma doença caracteristicamente estigmatizada e temida pelas mulheres, as quais, na maioria dos casos, associam a doença ao sofrimento e finitude de vida. No entanto, com os avanços científicos e tecnológicos voltados para o diagnóstico e tratamento da doença, somados às ações de promoção à informação e conhecimento sobre o câncer de mama, observa-se, cada vez mais, a relativização dessa visão estigmatizada sobre a doença, potencializando, assim, a autonomia dessas mulheres em busca da resolutividade ${ }^{30}$.

Entretanto, por intermédio da análise das falas das entrevistadas, tornou-se perceptível, em alguns momentos, que muitas mulheres que enfrentam essa doença tinham a sua autonomia coibida. Temos, portanto, as chamadas "pessoas-cordeiro", as quais, segundo Seixas ${ }^{27}$ e colaboradores, são aquelas caracteristicamente dóceis, obedientes, de fácil controle e manejo. Na maioria dos casos, as usuárias que possuem esse perfil são as mais aceitas por parte da equipe de saúde, tendo em vista a não relutância em aceitação sobre as práticas que thes são atribuídas. Profissionais que assumem tal linha de pensamento, presente no modelo hospitalocêntrico, impossibilitam que as usuárias reflitam sobre as práticas adotadas, os quais assumem a responsabilidade pela prescrição do modo como o outro deve trilhar a sua vida. Tal atitude pode ser percebida na seguinte fala:

Trabalhei [como cabeleireira] e até nos periodos antes de fazer a quimio. Aí ela disse assim [a médica]: você só vai ser R. até o dia da quimio, do dia da quimio pra lá, não vai pegar mais em nada, não pode fazer mais nada [...] (Ent.1).

No entanto, nem sempre haverá “pessoascordeiros" nos espaços coletivos, existem aquelas que decidem não mais serem coadjuvantes no seu processo saúde-doença, as quais revelam-se mulheres ativas, em busca da sua resolutividade. Dessa forma, a autonomia em saúde viabiliza a liberdade das cidadãs em gerir livremente as suas vidas, efetuando, racionalmente, as suas próprias escolhas ${ }^{27}$. Podemos perceber tal afirmativa na fala a seguir: 
Quando eu vim pra cá, que disse que não era o meu caso, eu poderia ficar em casa de boa, curtindo, levar minha vida! Mas, não! Eu fui pra outro médico! (Ent. 6).

Operar esse conceito na complexidade do cotidiano do trabalho em saúde não é simples. Sendo assim, vale salientar que o enfoque desta discussão não reside em desvalidar ou desqualificar as ações dos trabalhadores ou estigmatizar negativamente as suas práticas. Porém, é necessário evidenciar a necessidade de reflexão sobre a importância de haver uma construção coletiva do projeto terapêutico com a participação efetiva da usuária em relação ao caminho trilhado a ser escolhido ${ }^{27}$.

Mais uma vez, evidenciam-se a importância e a urgência em reavaliar e reformular as práticas do serviço e a formação dos futuros trabalhadores, incutindo a perspectiva de integralidade, em que 0 cuidado engloba as diferentes necessidades, valoriza a autonomia e é compatível com a realidade social e afetiva de cada sujeito ${ }^{8}$.

\section{CONCLUSÃO}

A mulher diagnosticada com câncer de mama possui diversas necessidades que não se concentram apenas na esfera fisiológica, mas perpassam as esferas psicológica e social. Dessa forma, é possivel afirmar que é necessária a priorização da produção do cuidado por meio do acolhimento e construção de vínculos (tecnologias-leves do cuidar) como estratégias a serem adotadas pelos pontos de atenção com as demais tecnologias a serem ofertadas, de acordo com as necessidades. Somado a isso, também é fundamental um amplo debate sobre os processos formativos no campo da saúde, além de uma discussão referente ao ato de cuidar em saúde no serviço, em que devem ser priorizadas as estratégias que permitam ações humanizadas e respeitosas aos modos de existência da vida dessas mulheres.

Para além disso, o estudo também demonstrou que a linha de cuidado para com as usuárias ainda se apresenta fragmentada, instável e com déficit importante em sua integração, o que pode ser solucionado com políticas que garantam um acesso universal e equânime, por meio de uma rede de atenção que garanta fluxos/caminhos resolutivos no sistema de saúde.

\section{CONTRIBUIÇÃO DOS AUTORES}

Marcio Costa de Souza e Milene Pereira de

Souza Santos contribuíram com o delineamento, a realização da pesquisa, a redação do manuscrito e a revisão crítica do manuscrito. Juliana Costa dos Santos Borges, Kamila Freitas Trindade, Evelin Duarte Serpa e Bruna Pereira Neves contribuíram com a realização da pesquisa e a redação do manuscrito.

\section{REFERÊNCIAS}

1. Instituto Nacional de Câncer José Alencar Gomes da Silva. Estimativa 2020: incidência de câncer no Brasil. Rio de Janeiro: INCA; 2019.

2. Global Câncer Observatory. Taxas estimadas de incidência padronizadas por idade (Mundial) em 2020, em todo o mundo, ambos os sexos, todas as idades. Lyon: International Agency for Research on Cancer; 2018.

3. Nascimento AS, Mello EVSL, Schneider LCL, Almeida FLA. Principais tratamentos utilizados no combate ao câncer de mama: uma revisão de literatura. Arquimudi [serial on the internet]. 2019 [cited 2021 0ut 15]; 23(3):201219. Available from: https://periodicos.uem.br/ ojs/index.php/ArqMudi/article/view/51538

4. Barros AES, Conde CR, Lemos TMR, Kunz JA, Ferreira MLSM. Sentimentos vivenciados por mulheres ao receberem o diagnóstico de câncer de mama. Rev enferm UFPE on line [serial on the internet]. 2018 [cited 2021 0ut 15]; 12(1):102111. Available from: https://periodicos.ufpe.br/ revistas/revistaenfermagem/article/view/23520

5. Instituto Nacional de Câncer José Alencar Gomes da Silva. Coordenação Geral de Prevenção e Vigilância. A mulher e o câncer de mama no Brasil. Divisão de Detecção Precoce e Apoio à Organização de Rede. Rio de Janeiro: INCA; 2018.

6. Sena L, Neves MGC. Os impactos psicológicos do diagnóstico e tratamento do câncer de mama em mulheres Com. Ciências Saúde [serial on the internet]. 2020 [cited 2021 0ut 18]; $30(01): 19$ 28. Available from: http://www.escs.edu.br/ revistaccs/index.php/comunicacaoemciencias d a s a u d e/article/view/367

7. Joaquín-Mingorance M, Arbinaga F., CarmonaMárquez J, \& Bayo-Calero J. Estratégias de enfrentamento e autoestima em mulheres com câncer de mama. Annals of Psychology [serial on the internet]. 
2019 [cited 2021 0ut 15]; 35(2):188-194. Available from: https://scielo.isciii.es/scielo.php?script=sci arttext\&pid $=$ S0212-97282019000200003

8. Souza MC; Borges JCS, Santos RS, Brito VCSG, Souza JN, Quintana RAC, et al. Itinerários terapêuticos de pessoas com doenças respiratórias crônicas. Rev. Eletrônica Acervo Saúde [serial on the internet]. 2020 [cited 2021 0ut 15]; 12(11):e4096. Available from: https://acervomais.com.br/index. $\mathrm{php/saude/article/view/4096}$

9. Costa JP, Jorge MSB, Vasconcelos MGF, Paula ML, Bezerra IC. Resolubilidade do cuidado na atenção primária: articulação multiprofissional e rede de serviços. Divulg. saúde debate [serial on the internet]. 2014 [cited 2021 0ut 15]; 38(103):733743. Available from: https://doi.org/10.5935/0103$\underline{1104.20140067}$

10. Rosa RB, Weis Pelegrini AHW, Lima MADS. Resolutividade da assistência e satisfação de usuários da Estratégia Saúde da Família. Rev. gaúcha enferm. [serial on the internet]. 2011 [cited 2021 0ut 15]; 32(2):345-351. Available from:https://www.scielo.br/j/rgenf/a/ SY5KhYJQg6XzPsZpfDM6P5Q/?lang=pt

11. Minayo MCS. Amostragem e saturação em pesquisa qualitativa: consensos e controvérsias. Rev. Pesquisa Qualitativa [serial on the internet]. 2017 [cited 2021 0ut 19]; 5(7):1-12. Available from: https:// editora.sepq.org.br/rpq/article/view/82

12. Souza MC, Souza JN. Access, Care, Social Inequalities and The Pandemic COVID 19 In Brazil. Biomed J Sci \& Tech Res [serial on the internet]. 2020 [cited 2021 0ut 15]; 31(4):2432724329. Available from: https://doi.org/10.26717/ BJSTR.2020.31.005125

13. Castro AMM, Silva JS, Macedo LCSA, Rosa NSF, Bertussi DC, Santos MLM, et al. Barreiras ao acesso a serviços de saúde à pessoa com deficiência no Brasil: uma revisão integrativa. Práticas e Cuidado: Revista de Saúde Coletiva [serial on the internet]. 2021 [cited 2021 Out 15]; 2:e11351. Available from: https://revistas.uneb.br/index.php/ saudecoletiva/article/view/11351

14. Hadad ACAC, Jorge A0. Continuidade do cuidado em rede e os movimentos de redes vivas nas trajetórias do usuário-guia. Divulg. saúde debate [serial on the internet]. 2018 [cited 2021 Out 15]; 42(spe4):198-210. Available from: https://www.scielo.br/j/sdeb/a/qKYndc8VcGL3T gjpRKQDS6w/?lang $=p t$

15. Ferreira TPS, Costa CT. Saúde e redes vivas de cuidado integral na atenção básica: articulando ações estratégicas no território. Revisbrato [serial on the internet] 2017 [cited 2021 Out 15]; 1(3):269281. Available from: https://revistas.ufrj.br/index. php/ribto/article/view/4750
16. Oliveira SB, Soares DA. Acesso ao cuidado do câncer de mama em um município baiano: perspectiva de usuárias, trabalhadores e gestores. Divulg. saúde debate [serial on the internet]. 2020 [cited 2021 Out 19]; 44(124):169-181. Available from: https:// doi.org/10.1590/0103-1104202012412

17. Guimarães AS, Velloso CS, Pereira ML, Viana TST. Prevenção e detecção precoce do câncer de mama na atenção primária à saúde: revisão integrativa. Brazilian Journal of Surgery and Clinical Research [serial on the internet]. 2020 [cited 2021 out 19]; 32(3):84-88. Available from: https://www. mastereditora.com.br/periodico/20201106 103604. pdf

18. Santos LMVR, Santos MPS, Merces MC, Souza JN, Souza MC. Barreiras de acesso em mulheres que vivem com câncer de mama. Revista Unilus Ensino e Pesquisa [serial on the internet]. 2021 [cited 2021 0ut 19]; 18(50):26-35. Available from: http://revista.unilus. edu.br/index.php/ruep/article/view/1380

19. Hadad ACAC. Jorge AO, Melo EM. A construção de redes vivas no percurso de usuários e familiares, vítimas de trauma, em sua trajetória na busca pelo cuidado. Rev. méd. Minas Gerais [serial on the internet]. 2016 [cited 2021 0ut 19]; 26 (Supl 8):S107-S111. Available from: $\quad$ http://rmmg.org/artigo/detalhes/2132

20. Menezes ELC, Scherer MDA, Verdi MI, Pires DP. Modos de produzir cuidado e a universalidade do acesso na atenção primária à saúde. Saúde e Soc [serial on the internet]. 2017 [cited 2021 0ut 19]; $26(4): 888$ 903. Available from: https://www.scielo.br/j/ sausoc/a/MxGqmyYkCy9yjkVnpNz3VbP/? lang=pt

21. Vasconcelos MIO, Xavier ALC, Nascimento MN, Cavalcante YA, Rocha SP, Gomes JS. Avaliação da resolutividade e efetividade da atenção primária à saúde: revisão integrativa de literatura. Sanare (Sobral, Online) [serial on the internet]. 2018 [cited 2021 Out 19]; 17(01):65-73. Available from: https:// sanare.emnuvens.com.br/sanare/article/view/1224

22. Quintana RAC, Souza ICS, Pereira JM, Pires RA, Lima RSGS, Merces MC, et al. Production of Interprofessional Care for the Person with Oncological Disease: A Study on the Patient's Perspective. Biomed J Sci \& Tech Res [serial on the internet]. 2020 [cited 2021 0ut 19]; 29(3): 22413-22418. Available from: https://biomedres.us/ fulltexts/BJSTR.MS.ID.004797.php

23. Merhy EE, Feuerwerker LCM. Novo olhar sobre as tecnologias de saúde: uma necessidade contemporânea. In: Merhy EE, Baduy RS, Seixas CT, Almeida DS, Slomp Junior H, organizadores. Avaliação compartilhada do cuidado em saúde: surpreendendo 0 instituído nas redes. Rio de Janeiro: Hexis; 2016. p. 59-72. 
24. Grabois V. Gestão do cuidado. In: Gondim R, Grabois V, Mendes Junior WV, organizadores. Qualificação dos Gestores do SUS. Rio de Janeiro (RJ): Fiocruz/ENSP/EAD; 2011. p. 153-190.

25. Sabino LMM, Brasil DRM, Caetano JA, Santos MCL, Alves MDS. Uso de tecnologia leve-dura nas práticas de enfermagem: análise de conceito. Aquichan [serial on the internet]. 2016 [cited 20210 ct 15]; 16(2):230239. Available from: http://www.scielo.org.co/scielo. php?script $=$ sci arttext\&pid =S 1657 59972016000200010\&lng=en

26. Santos DS, Mishima SM. Merhy EE. Processo de trabalho na Estratégia de Saúde da Família: potencialidades da subjetividade do cuidado para reconfiguração do modelo de atenção. Ciênc. saúde coletiva [serial on the internet]. 2018 [cited 2021 Out 19]; 23(3):861-870. Available from: https:// www.scielo.br/j/csc/a/s9bmJspgCcykVW6gddLytdG/ ?lang $=\mathrm{pt}$

27. Seixas CT, Baduy RS, Cruz KT, Bortoletto MSS, Slomp Junior $H$, Merhy EE. 0 vínculo como potência para a produção do cuidado em Saúde: o que usuáriosguia nos ensinam. Interface comum. saúde educ. [serial on the internet]. 2019 [cited 2021 0ut 19]; 23:e170627. Available from: https://www.scielo. br/j/csc/a/s9bmJspgCcykVW6gddLytdG/?lang=pt

28. Paula SHB, Volochko A, Figueiredo R. Linha de cuidado de câncer de mama e de colo de útero: um estudo sobre referência e contra referência em cinco regiões de saúde de São Paulo, Brasil. BIS, Bol. Inst. Saúde. 2016; 17(2):146-165.

29. Goldman RE, Figueiredo EN, Fusione SM, Souza KMJ, Almeida AM, Gutiérrez MGR. Rede de Atenção ao Câncer de Mama: perspectiva de gestores da saúde. Rev Bras Enferm [serial on the internet]. 2019 [cited 2021 0ut 19]; 72(Suppl 1):274-81. Available from: https://www.scielo.br/j/reben/a/c7QzYYsSb8hLTYyD $\underline{\mathrm{T}} \mathrm{YCkpM} /$ ?lang $=$ en

30. Machado MX, Soares DA, Oliveira SB. Significados do câncer de mama para mulheres no contexto do tratamento quimioterápico. Physis: Rev. de Saúde Coletiva [serial on the internet]. 2017 [cited 2021 Out 19]; 27(3):433451. Available from: https://www.scielo.br/ $\mathrm{j} /$ physis/a/sDSBbmp7YKkmMftcrcx98Kt/?lang=pt
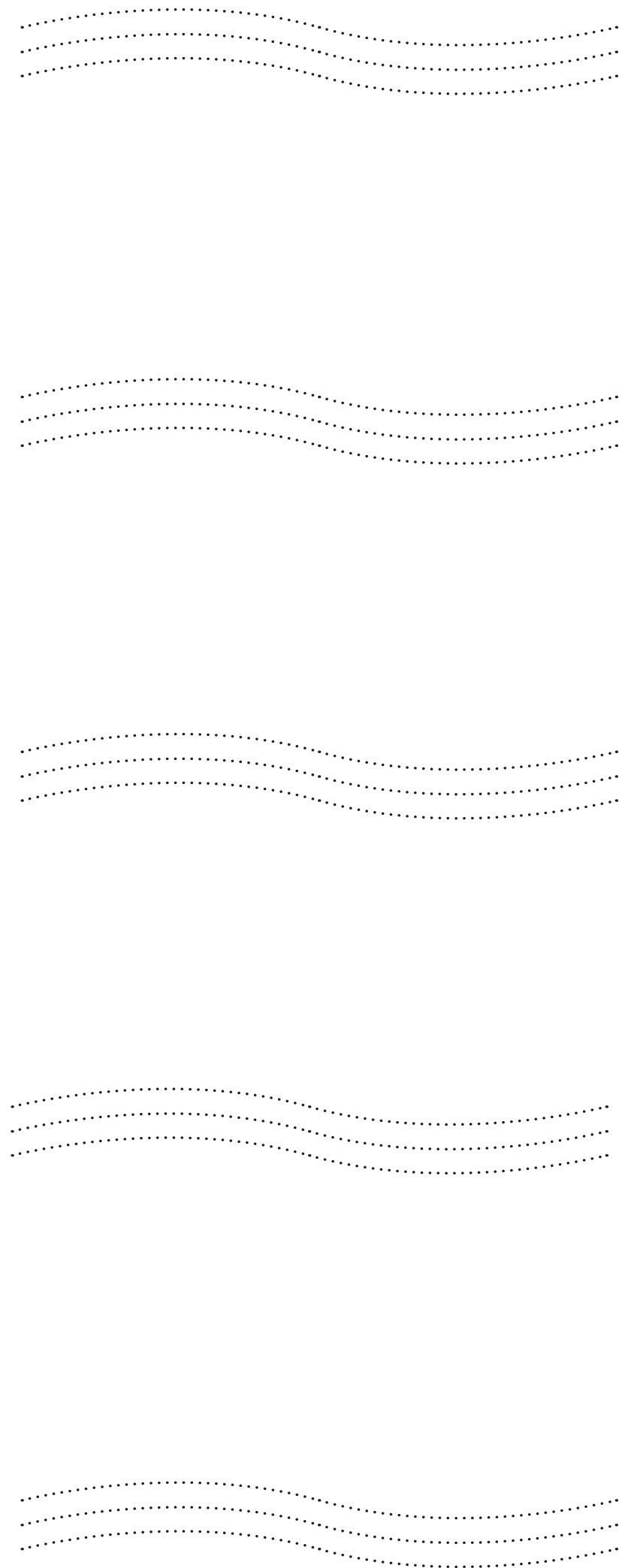